\title{
A Technique to Eliminate the Bounds in Nonlinear Programming Problems
}

\author{
ABHA Goel and G. C. Sharma \\ Department of Mathematics, Institute of Basic Science, \\ Agra University, Agra, India.
}

\begin{abstract}
In this paper, we consider the problem of minimization of an objective function having continuous first and second partial derivatives, subject to nonnegativity restrictions or upper and lower bounds on the variables of a nonlinear programming problem. An appropriate transformation of its variables has been made to convert such a constrained optimization problem into an unconstrained optimization problem. Some conditions are developed to guarantee that every local minimum of the unconstrained problem satisfies the Kuhn-Tucker's first order necessary conditions for a local minimum of the constrained problem. There are some conditions for which the transformed objective function maintains the convexity of the original objective function in a neighbourhood of the solution.
\end{abstract}

KEY WORDS : Transformation of variables. Bounds. Nonlinear programming problem, Convexity.

\section{Introduction}

The transformation techniques help in solving a nonlinear programming problem by reducing it to one or more unconstrained programs. Such a reduction is brought about by using a transformation that combines the objective function and the constraints. The advantage in using a transformation technique is that, problems without constraints are easier to be solved than those with constraints, because many techniques are available to solve the unconstrained programs.

In this paper, we consider a given nonlinear programming problem.

$$
\begin{array}{ll}
\text { Minimize } & f\left(y_{1}, y_{2}, \ldots, y_{n}\right) . \\
\text { Subject to } & y_{i} \geqslant 0 \quad, j=1,2, \ldots, m . \\
& l_{j} \leqslant y_{1} \leqslant u_{1}, \quad j=m+1, \ldots, n
\end{array}
$$


where $f$ is a nonlinear function having continuous first and second partial derivatives.

In 1966, Box ${ }^{[1]}$ presented some transformations that might be used to combine the objective function and the constraints.

Here, we take the transformation

$$
y_{j}=T_{j}\left(x_{j}\right), \quad j=1,2, \ldots, n
$$

Then the unconstrained problem is to

$$
\text { Minimize } F\left(x_{1}, x_{2}, \ldots, x_{n}\right)=f\left[T_{1}\left(x_{1}\right), T_{2}\left(x_{2}\right), \ldots, T_{n}\left(x_{n}\right)\right] .
$$

Now we have to solve problem (3), instead of solving the problem (1).

In section 2, we give some conditions that a transformation must satisfy in order, that it does not have any additional local minima for the unconstrained problem. In section 3 , we will give a criterion, i.e., to what extent, the transformation described in section 2 maintains convexity.

\section{Selection of an Appropriate Transformation}

Some conditions that the transformation (2) should satisfy are suggested by Powell ${ }^{[2]}$, which we call the global mapping conditions

(a) If $x_{j}$ is any real number, then $T_{j}\left(x_{j}\right)$ should satisfy the constraints on $y_{j}$.

(b) There should exist a value of $x_{j}$, for every feasible value of $y_{j}$ such that $y_{j}=T_{j}\left(x_{j}\right)$.

Following two cases are likely to occure:

\section{Case 1}

In some cases the global mapping conditions prevent the introduction of additional local minima into the problem. For Example

\section{Example 1}

Minimize $f\left(y_{1}, y_{2}\right)=\left(y_{1}-\alpha\right)^{2}+\left(y_{2}-\beta\right)^{2}$,

Subject to $y_{j} \geqslant 0, j=1,2$.

Solution is $y^{*}=(\alpha, \beta)^{T}, f\left(y^{*}\right)=0$.

Let $y_{j}=T_{j}\left(x_{j}\right)=\left(x_{j}^{2}-1\right)^{2} \geqslant 0, j=1,2$.

Global mapping conditions are satisfied by this tansformation and the transformed problem is :

Minimize $F\left(x_{1}, x_{2}\right)=\left(x_{1}^{4}-2 x_{1}^{2}+1-\alpha\right)^{2}+\left(x_{2}^{4}-2 x_{2}^{2}+1-\beta\right)^{2}$

which has four local minima and all of these map into $(\alpha, \beta)^{T}$, the solution of the original problem if $\alpha, \beta \leqslant 1$. Thus in this cae the global mapping conditions are sufficient in order that a transformation does not have any additional local minima for the unconstrained problem. 


\section{Case 2}

In some cases the global mapping conditions do not prevent the introduction of additional local minima into the problem. For example

\section{Example 2}

Minimize $\quad f\left(y_{1}, y_{2}\right)=\left(y_{1}-4\right)^{2}+\left(y_{2}-9\right)^{2}$

Subject to $y_{j} \geqslant 0, j=1,2$.

Solution is $y^{*}=(4,9)^{T}, f\left(y^{*}\right)=0$.

Let $y_{j}=T_{j}\left(x_{j}\right)=\left(x_{j}^{2}-1\right)^{2} \geqslant 0, j=1,2$.

Global mapping conditions are satisfied by this tansformation. The transformed problem is :

Minimize $F\left(x_{1}, x_{2}\right)=\left(x_{1}^{4}-2 x_{1}^{2}-3\right)^{2}+\left(x_{2}^{4}-2 x_{2}^{2}-8\right)^{2}$

which has nine local minima. Four of these map into $(4,9)^{T}$, the solution of the original problem, but remaining five $(0,0),(0, \pm 2),( \pm \sqrt{3}, 0)$ do not map into local minimum of $f$.

Here we see that there are some additional local minima in the solution of the transformed unconstrained problem. We require that any local minimum of the transformed problem (3) should satisfy the first order necessary conditions (KuhnTucker's) for a local minimum of the original problem (1). We find the following conditions on $T_{j}, j=1,2, \ldots, n$, are sufficient to this purpose which we call the local mapping conditions

(a) $T_{j}$ are functions with continuous first and second partial derivatives, where $j=1,2, \ldots, n$.

(b) $T_{j}\left(x_{j}\right) \geqslant 0$ for $j=1,2, \ldots, m$.

Also, $\quad T_{j}^{\prime}\left(x_{j}\right)=0 \Rightarrow T_{j}\left(x_{j}\right)=0$ and $T_{j}^{\prime}\left(x_{j}\right)>0$.

(c) $l_{j} \leqslant T_{j}\left(x_{j}\right) \leqslant u_{j}$ for $j=m+1, \ldots, n$.

Also, $T_{j}^{\prime}\left(x_{j}\right)=0 \Rightarrow$ either $T_{j}\left(x_{j}\right)=l_{i}$ and $T_{j}^{\prime}\left(x_{j}\right)>0$

or $T_{j}\left(x_{j}\right)=u_{j}$ and $T_{j}^{\prime}\left(x_{j}\right)<0$.

The following theorem is the required faithfulness of the mapping.

\section{Theorem}

Suppose $T_{j}, j=1,2 \ldots, n$, satisfy the local mapping conditions. Let $x$ be a stationary point of $F$ for which $\nabla^{2} F(x)$ is positive semidefinite. Then, for a local minimum of the original problem (1), $y=T(x)$ satisfies the first order necessary conditions, where $x=\left(x_{1}, x_{2}, \ldots, x_{n}\right)^{T}$ and $y=\left(y_{1}, y_{2}, \ldots, y_{n}\right)^{T}$ are the point in $n$-dimensional Euclidean space and $T(x)=\left[T_{1}\left(x_{1}\right), T_{2}\left(x_{2}\right), \ldots, T_{n}\left(x_{n}\right)\right]^{T}$. 


\section{Proof}

$$
\text { Since } \nabla F(x)=\left[f_{1} T_{1}^{\prime}\left(x_{1}\right), f_{2}^{\prime} T_{2}^{\prime}\left(x_{2}\right), \ldots, f_{n} T_{n}^{\prime}\left(x_{n}\right)\right]^{T}
$$

where $f_{j}^{a}$ denotes the first partial derivative of $f$ with respect to $y_{j}$.

If $x$ is stationary point of $F$, then for each value of $j$, where $j=1,2, \ldots, n$.

$$
\text { either } f_{i}^{\prime}=0 \text { or } T_{i}^{\prime}\left(x_{j}\right)=0
$$

By the local mapping conditions, if $T_{j}^{\prime}(x)=0$ then $y_{j}=T_{j}\left(x_{j}\right)$ lies on a boundary of the constraints of (1). It can be shown that the first order necessary conditions for a local minimum of the original problem (1) will be satisfied by a stationary point $F$ if and only if

and

$$
f_{j}^{\prime} T(x) \geqslant 0 \text { for } j=1,2, \ldots, m
$$

and

and

$$
\begin{aligned}
& \text { if, for } j=m+1, \ldots, n, \\
& f_{j}^{\prime} T(x) \geqslant 0 \quad \text { when } y_{l}=l_{j} \\
& f_{j}^{\prime} T(x) \geqslant 0 \text { when } y_{j}=u_{j}
\end{aligned}
$$

By assumption, we are at a stationary point of $F$ at which the Hessian of $F$ is positive semidefinite. We will now show that this automatically guarantees that the conditions (5) on $f_{j}^{\prime}$ hold.

It can be seen that

$$
\begin{array}{ll} 
& \nabla^{2} F(x)=D \nabla^{2} f(y) D+E \\
\text { where } & D=\operatorname{diag}\left\{T_{j}^{\prime}\left(x_{j}\right): j=1,2, \ldots, n\right\}, \\
\text { and } & E=\operatorname{diag}\left\{f_{j}^{\prime} T_{j}^{\prime \prime}\left(x_{j}\right): j=1,2, \ldots, n\right\}
\end{array}
$$

At a stationary point $x$, if $\nabla^{2} F(x)$ is positive semidefinite, then $f_{j}^{\prime} T_{j}^{\prime \prime}\left(x_{j}\right) \geqslant 0$ for any value of $j$ for which $T_{j}^{\prime}\left(x_{j}\right)=0$.

$$
\text { By }(4) \text {, if } T_{j}^{\prime}\left(x_{j}\right) \neq 0, \text { then } f_{j}^{\prime}=0 \text { for } j=1,2, \ldots, n \text {. }
$$$$
\text { If } T_{j}^{\prime}\left(x_{j}\right)=0 \text {, then by the local mapping conditions, }
$$

and

$$
\begin{aligned}
& f_{j}^{\prime} T_{j}^{\prime \prime}\left(x_{j}\right) \geqslant 0 \Rightarrow f_{j}^{\prime} \geqslant 0 \text { for } j=1,2, \ldots, m, \\
& f_{j}^{\prime} T_{j}^{\prime \prime}\left(x_{j}\right) \geqslant 0 \Rightarrow \text { either } y_{j}=l_{j} \text { and } f_{j}^{\prime} \geqslant 0
\end{aligned}
$$$$
\text { or } y_{j}=u_{j} \text { and } f_{j}^{\prime} \geqslant 0 \text { for } j=m+1, \ldots, n \text {. }
$$

These are the first order necessary conditions.

The local mapping conditions guarantee that any local minimum of (3) satisfies the first order necessary conditions for a local minimum of (1) but these conditions have to guarantee that a solution of (3) can actually be found. For example

\section{Example 3}

$$
\text { Minimize } \quad f\left(y_{1}, y_{2}\right)=y_{1}+2 y_{2}
$$


Subject to

$$
-1 \leqslant y_{j} \leqslant 1, \quad j=1,2
$$

The solution $y^{*}=(-1,-1)^{T}$ and $f\left(y^{*}\right)=-3$

Let $y_{j}=T_{j}\left(x_{j}\right)=\frac{2 x_{\mathrm{j}}}{x_{j}^{2}+1}, j=1,2$

Local and global mapping conditions are satisfied by this transformation and the transformed problem is

$$
\text { Minimize } F\left(x_{1}, x_{2}\right)=\frac{2 x_{1}}{x_{1}^{2}+1}+\frac{4 x_{2}}{x_{2}^{2}+1}
$$

We use steepest descent method (see Flecther ${ }^{[3]}$ or Powell[2]) to solve the transformed problem,

$$
\nabla F\left(x_{1}, x_{2}\right)=\left[\frac{2-2 x_{1}^{2}}{\left(x_{l}^{2}+1\right)^{2}}, \frac{4-4 x_{2}^{2}}{\left(x_{2}^{2}+1\right)^{2}}\right]^{T}
$$

Suppose we begin with $x_{1}^{(1)}>1, x_{2}^{(1)}>1$, then $\nabla F\left(x_{1}^{(1)}, x_{2}^{(2)}\right)$ will have both of its components negative, and then the next point $\left(x_{1}^{(2)}, x_{2}^{(2)}\right)$ will be further away from the solution comparative to point $\left(x_{1}^{(1)}, x_{2}^{(1)}\right)$.

In this case both of the global and local mapping conditions are satisfied by the transformation but we have no solution of the transformed problem. To avoid this problem, we give some conditions that a transformation should satisfy, which we call the complete mapping conditions

(a) The functions $T_{j}$ should satisfy the global and local mapping conditions.

(b) If $\lim _{x \rightarrow \pm \infty} T_{j}\left(x_{j}\right)=K$, then

$$
\begin{aligned}
& \text { If } j=1,2, \ldots, m \text { then } K=0 \\
& \text { If } j=m+1, \ldots, n \text { then } K=l_{j} \text { or } K=u_{j}
\end{aligned}
$$

For Example 3, we find that an appropriate transformation is $y_{j}=T_{j}\left(x_{j}\right)=\sin x_{j}$, $j=1,2$

When we use the periodic transformations, we must take certain precautions. A particular interval in the domain of the function should be designated as the basic part of the function, and any values generated which fall outside of this interval should be displaced back into it.

We give below some appropriate transformations to some specific constraints.

(1) If we have the constraint $y_{i} \geqslant 0$, then any one of the following transformations can be used
(a) $y_{i}=x_{i}^{2}$
(b) $y_{i}=e^{x_{i}}$
(c) $y_{i}=\left|x_{i}\right|$

(2) If we have the constraint $0 \leqslant y_{i} \leqslant 1$, then any one of the following transfor- 
mations can be used

$$
\begin{aligned}
& y_{i}=\sin ^{2} x_{i} \\
& y_{i}=\frac{e^{x_{i}}}{e^{x_{i}}+e^{-x_{i}}}
\end{aligned}
$$

(3) If we have the constraint $l_{i} \leqslant y_{i} \leqslant u_{i}$, then the following transformation can be used

$$
y_{i}=l_{i}+\left(u_{i}-l_{i}\right) \sin ^{2} x_{i}
$$

(4) If we have the constraint $-1 \leqslant y_{i} \leqslant 1$, then the following transformation can be used

$$
y_{i}=\sin x_{i}
$$

(5) If we have the constraint $y_{1}^{2}+y_{2}^{2} \leqslant 1$, then the following transformation can be used

$$
y_{1}=\sin x_{1} \sin ^{2} x_{2}, y_{2}=\cos x_{1} \sin ^{2} x_{2}
$$

(6) If we have the constraint $0 \leqslant y_{i} \leqslant y_{i} \leqslant y_{k}$, then the following transformation can be used

$$
y_{i}=x_{i}^{2}, y_{j}=x_{i}^{2}+x_{j}^{2}, \quad y_{k}=x_{i}^{2}+x_{j}^{2}+x_{k}^{2}
$$

\section{Discussion of Convexity}

Convexity of a problem is an important feature in the solution of the problem. Here we assume that the original objective function $f$ is convex and we have to determine the convexity of the transformed objective function $F$. Unfortunately, the following examples illustrate that although the original objective function $f$ is convex, the transformed objective function is not necessarily convex.

\section{Example 4}

$$
\begin{array}{ll}
\text { Minimize } & f\left(y_{1}, y_{2}\right)=\left(y_{1}+3\right)^{2}+\left(y_{2}-4\right)^{2} \\
\text { Subject to } & y_{1}, y_{2} \geqslant 0 \\
\text { The solution is } & (0,4)^{T} \text { and } f\left(y^{*}\right)=9
\end{array}
$$

Let $y_{j}=T_{j}\left(x_{j}\right)=x_{j}^{2} \geqslant 0, j=1,2$

Then the transformed problem is

$$
\begin{array}{ll} 
& F\left(x_{1}, x_{2}\right)=\left(x_{1}^{2}+3\right)^{2}+\left(x_{2}^{2}-4\right)^{2} \\
& \nabla^{2} f\left(y_{1}, y_{2}\right)=\left[\begin{array}{cc}
2 & 0 \\
0 & 2
\end{array}\right] \\
\text { and } & \nabla^{2} F\left(x_{1}, x_{2}\right)=\left[\begin{array}{cc}
12 x_{1}^{2}+12 & 0 \\
0 & 12 x_{2}^{2}-16
\end{array}\right]
\end{array}
$$

Here we see that although the original objective function $f$ is strictly convex for all values of $y$, the transformed objective function $F$ is strictly convex only in a neighbourhood of the solution. 


\section{Example 5}

Minimize

$$
f\left(y_{1}, y_{2}\right)=4 y_{1}^{2}-3 y_{1} y_{2}+4 y_{2}^{2}
$$

Subject to

$$
y_{1}, y_{2} \geqslant 0
$$

The solution is $y^{*}=(0,0)^{T}$ and $f\left(y^{*}\right)=0$

Let $y_{j}=T_{j}\left(x_{j}\right)=x_{j}^{2} \geqslant 0, j=1,2$

Then the transformed problem is

$$
\begin{array}{ll}
F\left(x_{1}, x_{2}\right)=4 x_{1}^{4}-3 x_{1}^{2} x_{2}^{2}+4 x_{2}^{4} \\
\text { and } \quad \nabla^{2} f\left(y_{1}, y_{2}\right)=\left[\begin{array}{cc}
8 & -3 \\
-3 & 8
\end{array}\right] \\
\nabla^{2} F\left(x_{1}, x_{2}\right)=\left[\begin{array}{cc}
48 x_{1}^{2}-6 x_{2}^{2} & -12 x_{1} x_{2} \\
-12 x_{1} x_{2} & 48 x_{2}^{2}-6 x_{1}^{2}
\end{array}\right]
\end{array}
$$

The eigenvalues of $\nabla^{2} f\left(y_{1}, y_{2}\right)$ are 5 and 11 and we see that although the original objective function $f$ is strictly convex for all values of $y$, the transformed objective function $F$ is not convex.

We can see that every neighbourhood of the solution $(0,0)$ contains a point of the form $(\delta, 0)$ for which $\nabla^{2} F(\delta, 0)=\left[\begin{array}{cc}48 \delta^{2} & 0 \\ 0 & -6 \delta^{2}\end{array}\right]$ then it is easy to see that the transformed objective function $F$ is not convex in any neighbourhood of the solution.

From Examples 4 and 5 we come to conclude that although the original objective functions are globally convex is both the examples, the transformed objective function $F$ is convex in a neighbourhood of the solution in Example 4 and it is not convex in any neighbourhood of the solution in Example 5.

Now, we give a distinction between the transformed problems, one type is convex in a neighbourhood of the solution and the other is not convex in any neighbourhood of the solution.

We do this by considering two types of solutions of our problems.

Suppose Example 4 illustrates a solution of the first kind, then

(1) $y=T(x)$ is a solution of the first kind if when $y_{j}$ lies on a boundary of the constraints of the original problem (1) then $f_{i}^{\prime}(y) \neq 0$.

(2) $y=T(x)$ is a solution of the second kind if when $y_{i}$ lies on a boundary of the constraints of the original problem (1) then $f_{i}^{\prime}(y)=0$ for at least one value of $i$.

\section{Applications}

The areas of application of such problems are quite many, particularly in industrial technology. To mention a few are agriculture, transportation, storage problems, ...etc. Also these problems arise in design of experiments, circuit design and least squares data fitting in cases where the variables are subject to bounds. The use of this technique has been found suitable to most of them. 


\section{Acknowledgement}

The authors are thankful to the learned referees for their fruitful suggestions.

\section{References}

[1] Box, M.J., A comparison of several current optimization methods and the use of transformations in constrained problems, The Computer Journal, 9: 67-77 (1966).

[2] Powell, M.J.D., Problems related to unconstrained optimization, in: Murray, W. (ed.), Numerical Methods for Unconstrained Optimization. New York. Academic Press (1972).

[3] Fletcher, R., A new approach to variable metric algorithms. The Computer Journal, 13: 317-322 (1970). 
طريقـة للتخلص من القيـود في نماذج الـبريجة غير الخطيـة

$$
\text { أبها جويل و جي · سي . شارما }
$$

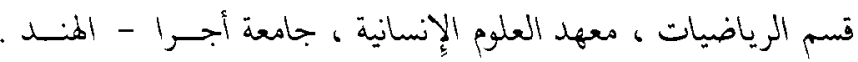

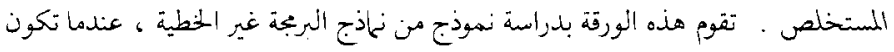

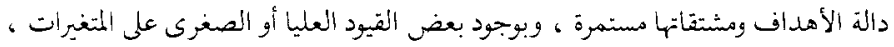

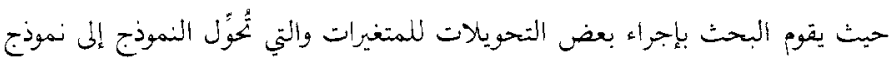

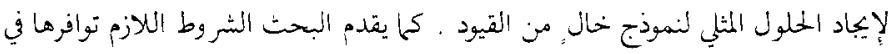

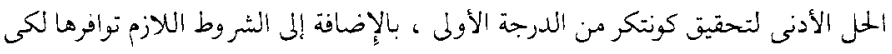

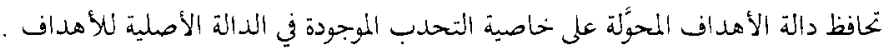

